\title{
Tsukushi is essential for the development of the inner ear
}

\author{
Toru Miwa ${ }^{1,2,3,4^{*}}$ (D), Kunimasa Ohta $5,6,7,8$, Naofumi Ito ${ }^{5}$, Satoko Hattori ${ }^{9}$, Tsuyoshi Miyakawa ${ }^{9}$, Toru Takeo ${ }^{10}$, \\ Naomi Nakagata ${ }^{10}$, Wen-Jie Song ${ }^{6,11}$ and Ryosei Minoda ${ }^{4}$
}

\begin{abstract}
Tsukushi (TSK) — a small, secreted, leucine-rich-repeat proteoglycan —interacts with and regulates essential cellular signaling cascades. However, its functions in the mouse inner ear are unknown. In this study, measurement of auditory brainstem responses, fluorescence microscopy, and scanning electron microscopy revealed that TSK deficiency in mice resulted in the formation of abnormal stereocilia in the inner hair cells and hearing loss but not in the loss of these cells. TSK accumulated in nonprosensory regions during early embryonic stages and in both nonprosensory and prosensory regions in late embryonic stages. In adult mice, TSK was localized in the organ of Corti, spiral ganglion cells, and the stria vascularis. Moreover, loss of TSK caused dynamic changes in the expression of key genes that drive the differentiation of the inner hair cells in prosensory regions. Finally, our results revealed that TSK interacted with Sox2 and BMP4 to control stereocilia formation in the inner hair cells. Hence, TSK appears to be an essential component of the molecular pathways that regulate inner ear development.
\end{abstract}

Keywords: Tsukushi, Hair cell stereocilia, Sox2, BMP4, Hearing

\section{Introduction}

Tsukushi (TSK) is a small, secreted leucine-rich-repeat proteoglycan and extracellular signaling molecule that participates in various developmental processes in vertebrates [1-3]. The diverse functions of TSK depend on its ability to bind and interact with various intermediate molecules of some major signaling pathways. TSK interactions with BMP, FGF, TGF- $\beta$, and Wnt signaling pathways have been previously reported $[1,4-6]$ and shown to affect the development of the central nervous networks $[7,8]$, as well as wound healing [9]. TSK is reportedly expressed in the chick retina $[3,10]$, the subventricular zone and hippocampus in the mouse

\footnotetext{
* Correspondence: t-miwa@kitano-hp.or.jp

'Department of Otolaryngology-Head and Neck Surgery, Kitano Hospital, Tazuke Kofukai Medical Research Institute, 2-4-20 Ougimaci, Kita-ku, Osaka 5308084, Japan

${ }^{2}$ Department of Otolaryngology-Head and Neck Surgery, Graduate School of Medicine, Kyoto University, 54 Shogoin Kawahara-cho, Sakyo-ku, Kyoto 6068507, Japan

Full list of author information is available at the end of the article
}

brain [8], and dermal hair follicles in mice [9]. However, TSK function and localization in the inner ear have not yet been investigated. Indeed, the molecular mechanisms underlying the inner ear development and acquisition of normal hearing are still unknown [11].

Accordingly, we studied TSK expression and function during inner ear morphogenesis and after birth. In addition, we investigated TSK interaction with BMP. BMP signaling is crucial for the development of the inner ear, and its gradient expression in the developing inner ear causes dysmorphogenesis of the inner ear [12, 13]. Therefore, after confirming TSK expression, we performed histological examination to investigate the phenotypes related to TSK deficiency, namely, hearing and changes in cell morphology. We then investigated the molecular mechanisms of TSK interaction with BMP4 by immunostaining and assessing mRNA expression levels. Finally, we investigated specific effects of TSK deficiency on stereocilia of the hair cells (HCs), while leaving the rest of the inner ear morphologically intact. 


\section{Results}

TSK was differentially expressed in various tissues during embryonic development

For the first time, we mapped TSK localization in the inner ear. To do this, embryos, in which the LacZ reporter gene was introduced within the coding exon of TSK, were fixed, cryosectioned, and stained with X-gal. At embryonic day 9.5 (E9.5) and E11.5, TSK-expressing cells were localized in the ventral otocyst tissues, which eventually differentiate into the cochlear duct (Fig. 1a, b). At E13.5 and E15.5, TSK-expressing cells were detected at the lateral wall of the cochlear duct, which would later differentiate into the stria vascularis and spiral ligament, and not to spiral ganglion cells (SGCs) (Fig. 1c-h). At postnatal stage 0 (P0), TSK-expressing cells were observed in some SGCs, the OC, inside and outside of the lateral wall that differentiates into the stria vascularis and spiral ligaments, and the greater epithelial ridge, which differentiates into the spiral limbus and tectorial membrane (Fig. 1i-k). At P10, TSK was expressed in some SGCs, the OC, the stria vascularis, and type I fibrocytes in spiral ligaments (Fig. 11-n). Finally, at P30, TSK was localized in SGCs, the OC, the stria vascularis, and type III fibrocytes in spiral ligaments (Fig. 1o-q).

\section{TSK deficiency caused hearing abnormalities}

At P30, frequency-averaged auditory brainstem response (ABR) thresholds for control (CONT) and TSK-knock out $(\mathrm{KO})$ mice were $29.0 \pm 5.83$ and $80.0 \pm 9.29 \mathrm{dBSPL}$ at
$4 \mathrm{kHz}, 25.0 \pm 9.48$ and $56.5 \pm 15.2 \mathrm{dBSPL}$ at $12 \mathrm{kHz}$, and $37.6 \pm 4.36$ and $61.7 \pm 14.3 \mathrm{dBSPL}$ at $20 \mathrm{kHz}$, respectively. Differences were significant at all frequencies (Fig. 2a; $p=0.002$ at $4 \mathrm{kHz}, p=0.002$ at $12 \mathrm{kHz}$, and $p=0.004$ at $20 \mathrm{kHz}$ ). On the other hand, levels of the frequencyaveraged distortion product of otoacoustic emission (DPOAE) were 9.08 \pm 3.34 and $10.5 \pm 6.4 \mathrm{dBSPL}$ at 4 $\mathrm{kHz}, 8.99 \pm 7.63$ and $10.8 \pm 4.22 \mathrm{dBSPL}$ at $5.6 \mathrm{kHz}$, $0.38 \pm 8.96$ and $1.6 \pm 7.66 \mathrm{dBSPL}$ at $8 \mathrm{kHz}, 3.77 \pm 12.9$ and $7.34 \pm 13.9 \mathrm{dBSPL}$ at $11.3 \mathrm{kHz},-2.70 \pm 15.7$ and $8.11 \pm 11.1 \mathrm{dBSPL}$ at $16 \mathrm{kHz}, 17.1 \pm 13.6$ and $22.4 \pm 10.7$ $\mathrm{dBSPL}$ at $22.6 \mathrm{kHz}$, and $1.35 \pm 9.99$ and $12.1 \pm 12.4$ dBSPL at $29 \mathrm{kHz}$, respectively. These differences were not statistically significant at all tested frequencies (Fig. $2 \mathrm{~b} ; p=0.29$ at $4 \mathrm{kHz}, p=0.27$ at $5.6 \mathrm{kHz}, p=0.38$ at $8 \mathrm{kHz}, p=0.29$ at $11.3 \mathrm{kHz}, p=0.06$ at $16 \mathrm{kHz}, p=$ 0.19 at $22.6 \mathrm{kHz}$, and $p=0.06$ at $29 \mathrm{kHz}$ ).

Cognitive function was evaluated using the fearconditioning test to clarify the hearing loss and to examine learning and memory function in the TSK$\mathrm{KO}$ mice. In this test, freezing responses between the CONT and TSK-KO mice in the conditioning phase (auditory stimulus and foot shock, 2-min interstimulus interval) or contextual testing (one day after conditioning for $300 \mathrm{~s}$ ) were not significantly different (Additional file 1, conditioning phase, $p=0.94$; contextual testing, $p=0.75$ ). Levels of freezing in the TSK-KO mice significantly decreased during the auditory cue in an altered context (auditory stimulus for

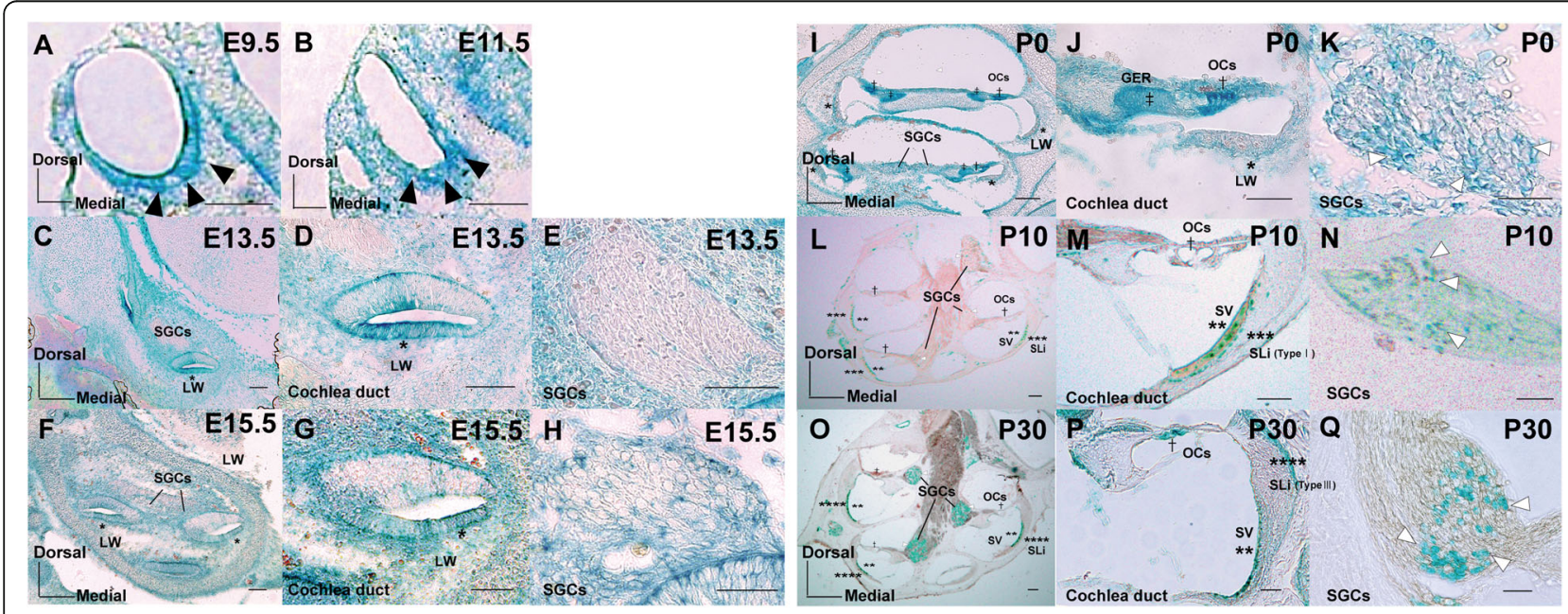

Fig. 1 Spatiotemporal expression of TSK in the inner ear. a, b At E9.5 and E11.5, TSK-expressing cells were localized in the ventral tissues of the otocyst, which would differentiate into the cochlear duct (black arrowheads). c-h At E13.5 and E15.5, TSK-expressing cells were localized at the LW of the cochlear duct, which would differentiate into SV (asterisks in $\mathbf{c}, \mathbf{d}, \mathbf{f}, \mathbf{g}$ ), and not SGCs (e, h). i-k At P0, TSK was expressed in some SGCs (white arrowheads in $\mathbf{i}, \mathbf{k}$ ), the OC (dagger in $\mathbf{i}, \mathbf{j}$ ), LW, which differentiates into the SV and SLi (asterisks in $\mathbf{i}$, $\mathbf{j}$ ), and in the GER, which differentiates into the spiral limbus and tectorial membrane (double dagger in $\mathbf{i}, \mathbf{j}$ ). I-n At P10, TSK was expressed in some SGCs (white arrowheads in $\mathbf{I}, \mathbf{n}$ ), the OC (dagger in I, $\mathbf{m}$ ), the SV (double asterisk in $\mathbf{I}, \mathbf{m}$ ), and type I fibrocytes of the SLi (triple asterisk in $\mathbf{I}$, $\mathbf{m}$ ). (o- $\mathbf{q})$ At P30, TSK was expressed in some SGCS (white arrowheads in $\mathbf{o}, \mathbf{q}$ ), the OC (dagger in $\mathbf{o}, \mathbf{p}$ ), the SV (double asterisk in $\mathbf{o}$, $\mathbf{p}$ ), and type III fibrocytes of the SLi (quattro asterisk in o, p). GER: greater epithelial ridge, LW: lateral wall, OC: organ of Corti, SGC: spiral ganglion cell, SV: stria vascularis, SLi: spiral ligament. Scale bars represent $100 \mu \mathrm{m}(\mathbf{a}$ and $\mathbf{b})$ or $50 \mu \mathrm{m}(\mathbf{c}-\mathbf{q})$ 


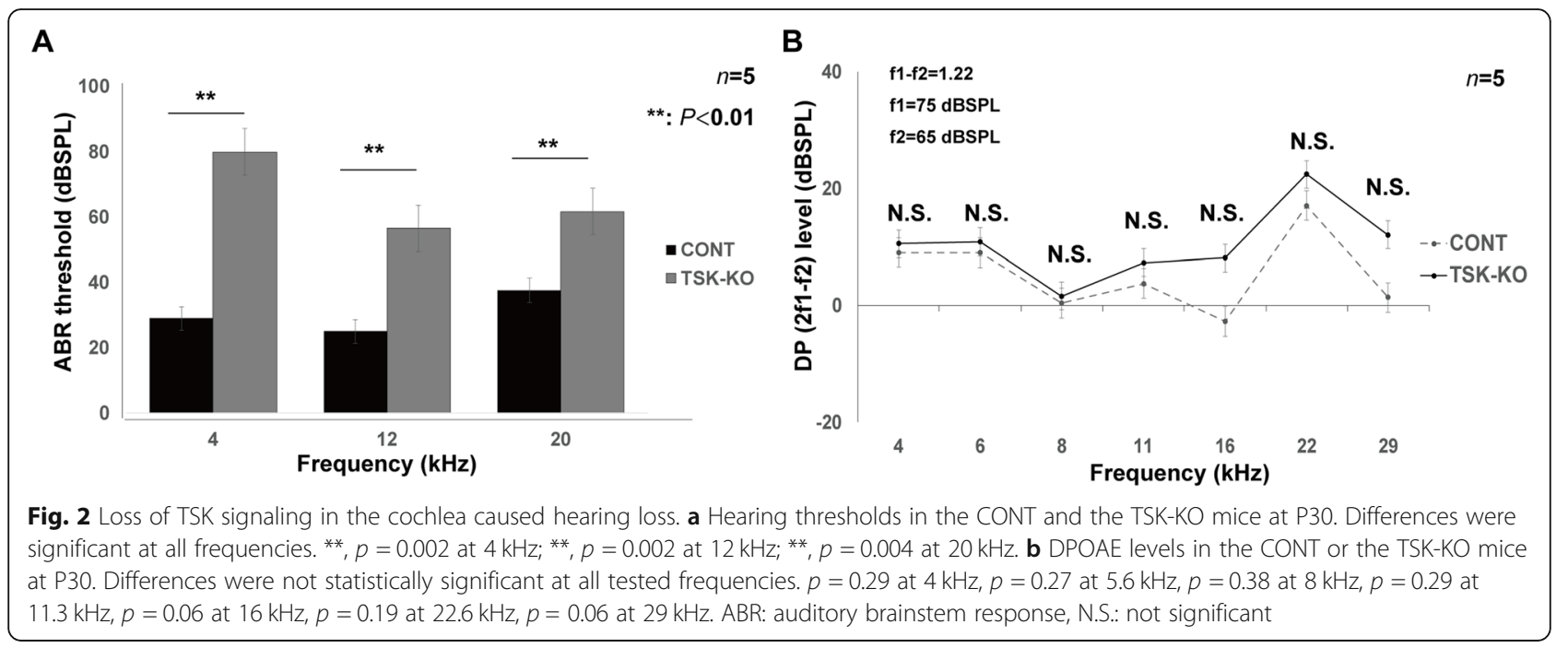

$180 \mathrm{~s})$ compared with that in the CONT mice (Additional file 1, $p=0.02$ ).

\section{TSK deficiency caused the formation of abnormal stereocilia, but not loss of hair cells}

Morphology of the HCs, revealed by Phalloidin staining and anti-Myo7a staining, appeared normal in the CONT and TSK-KO mice (Fig. 3a, b). For the quantitative analysis of the body of hair cells, we counted the number of
HCs. The numbers of the HCs at apical, middle, and basal turns were not significantly different (Fig. 3c) with $p=0.09,0.30$, and 0.18 , respectively, for the outer $\mathrm{HCs}$, and $p=0.46,0.29$, and 0.45 , respectively, for the inner HCs. However, stereocilia in the inner HCs were significantly shortened and dislocated in the TSK-KO mice (Fig. 3d-f, $p<0.0001$ for all turns), as visualized by scanning electron microscopy. Stereocilia in the outer HCs were unaffected. Stereocilia of inner HCs at P0 and P6

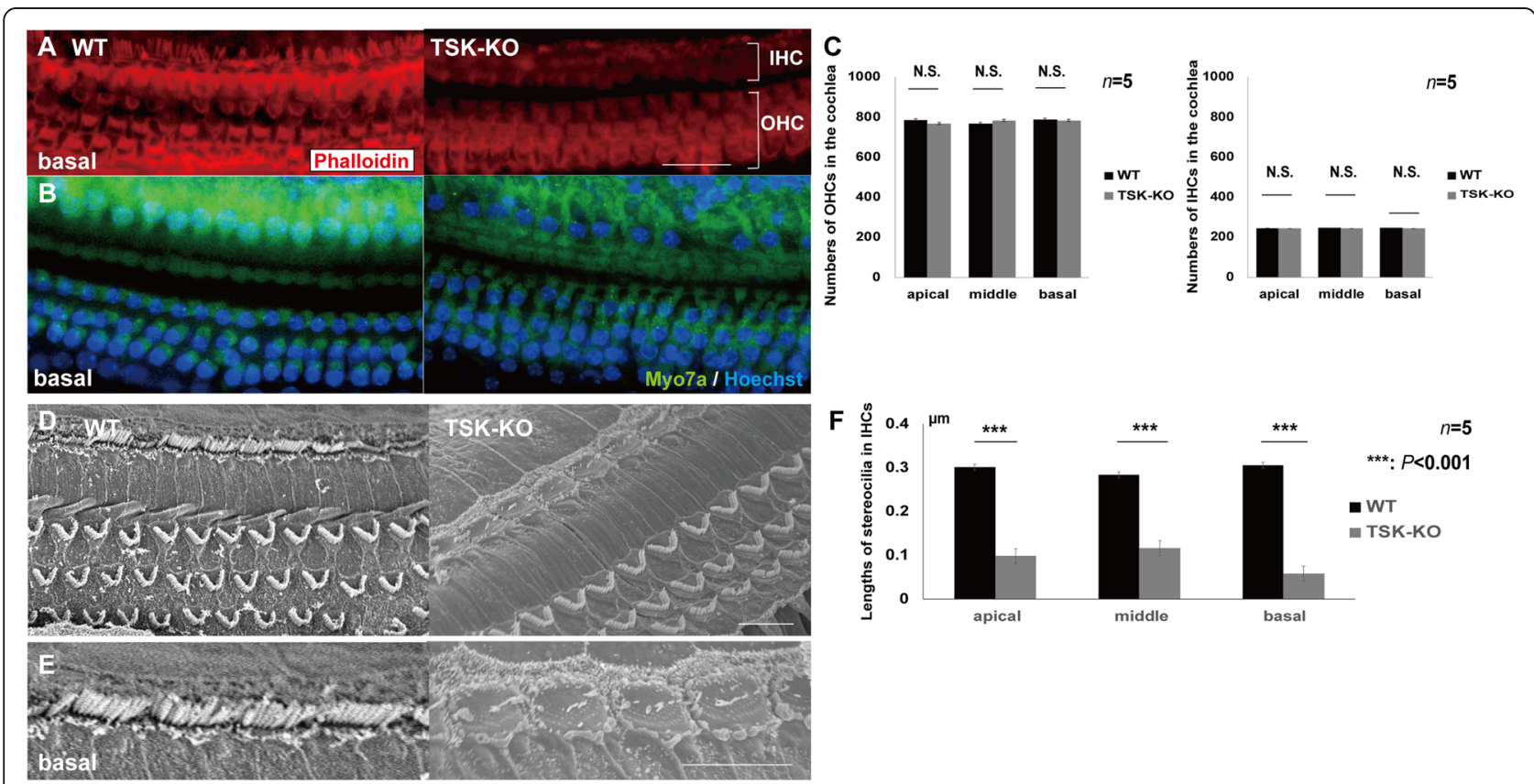

Fig. 3 Enumeration of the hair cells in surface preparations of cochleae at P30. a, b Phalloidin staining and immunostaining with anti-Myo7a of the hair cells at basal turns in the CONT (left) and TSK-KO mice (right). c The numbers of outer and inner hair cells in the CONT and TSK-KO mice were not significantly different; $p=0.09,0.30$, and 0.18, respectively, for apical, middle, and basal outer hair cells; $p=0.46,0.29$, and 0.45 , respectively, for apical, middle, and basal inner hair cells. d, e Scanning electron micrographs of the hair cells in the CONT and TSK-KO mice. $\mathbf{f}$ Length of stereocilia in the inner hair cells in the CONT and TSK-KO mice. Stereocilia in the inner hair cells were significantly shortened and dislocated in the TSK-KO mice. ${ }^{* * *}, p<0.0001$ at all turns. IHC: inner hair cell, OHC: outer hair cell, N.S.: not significant, WT: wild-type 
were likely to be shortened in the surface preparation; however, a quantitative analysis could not be performed from our results (Additional file 2). As HC development has been shown to be related to the expression and localization of Sox2, which is known to be regulated in other pathways by BMP4 via TSK, we next investigated the expression and localization of all these proteins at different developmental stages in the mouse.

\section{TSK loss suppressed Sox2 and redistributed BMP4}

We examined Sox 2 and BMP4 expression in the cochlea at various developmental stages in mice to assess differentiation of the HCs. Sox2 was detected by in situ hybridization and immunolabeling in prosensory regions in the CONT mice but was diminished in the TSK-KO mice at E13.5, E15.5, and P0 (Fig. 4a, b). Quantitative reverse-transcription polymerase chain reaction (qRTPCR) of the cochlear epithelial tissue revealed that Sox2 mRNA was also significantly diminished in the absence of TSK (Fig. 4d), with $p=0.03,0.04$, and 0.01 at E13.5, E15.5, and P0, respectively, as compared to CONT tissues. Similarly, BMP4 was detected by in situ hybridization in nonprosensory regions and outer sulcus in the CONT mice. In the TSK-KO mice, BMP4 was diminished in the outer sulcus but thinly distributed around the cochlear epithelium (Fig. 4c). Finally, qRTPCR of the cochlear epithelial tissue revealed that BMP4 mRNA increased in the TSK-KO, but only marginally (Fig. 4e), with $p=0.23,0.37$, and 0.19 at E13.5, E15.5, and P0, respectively. Considering these TSK-dependent, region-specific changes in Sox 2 and BMP4 expression, we next investigated changes in the cellular composition of the tissue.

\section{Spiral ganglion cells were diminished at the cochlear basal turn in the TSK-KO mice}

The average number of the SGCs in the CONT and TSK-KO mice was $100.6 \pm 10.1$ and $98.0 \pm 10.1$ at the apical turn, $126.4 \pm 12.8$ and $134.3 \pm 24.9$ at the middle turn, and $115.5 \pm 20.4$ and $88.6 \pm 25.2$ at the basal turn, respectively. Differences were significant at the basal turn (Fig. 5a, b) with $p=0.39,0.15$, and 0.02 for the apical, middle, and basal turn, respectively.

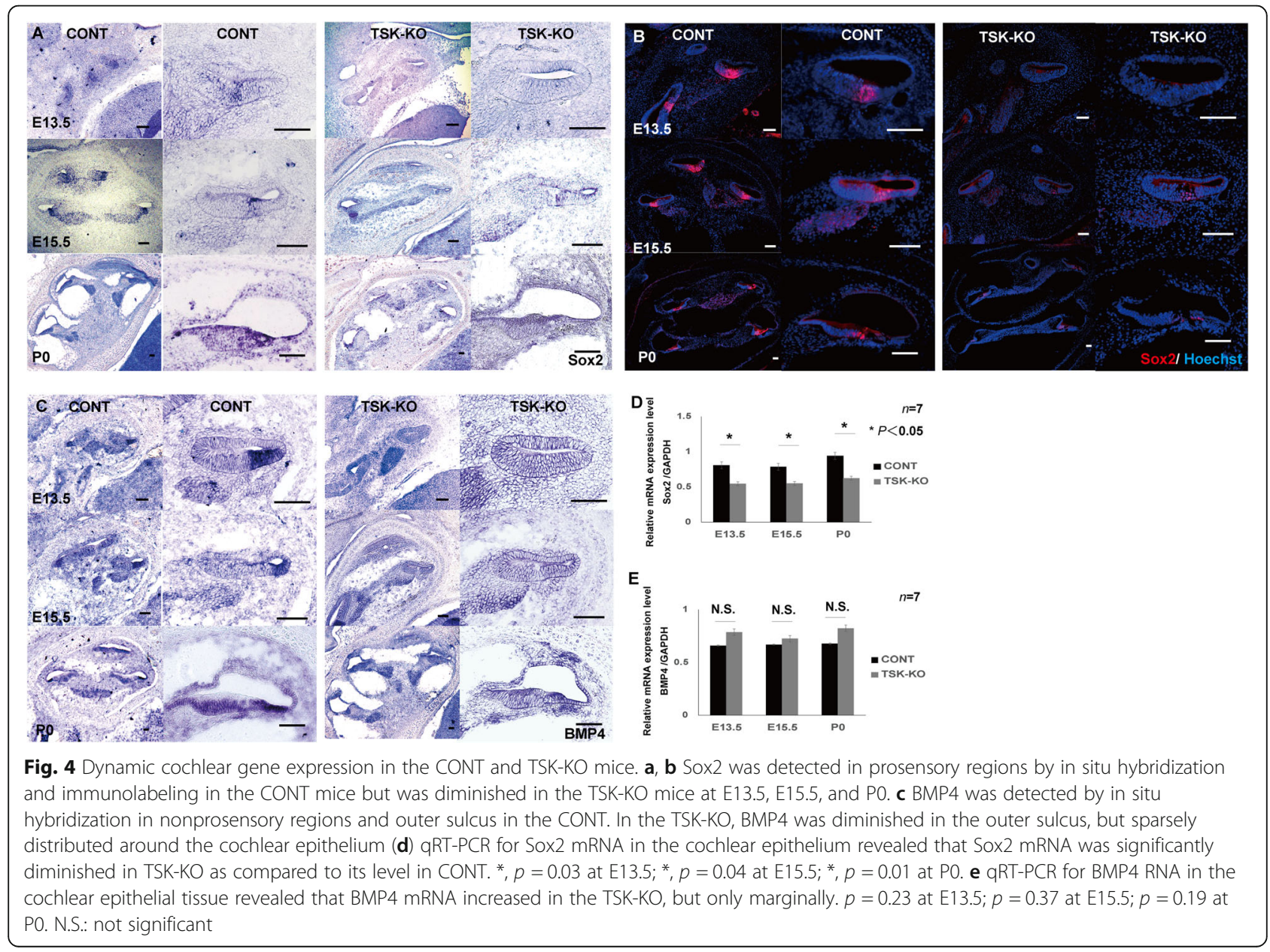




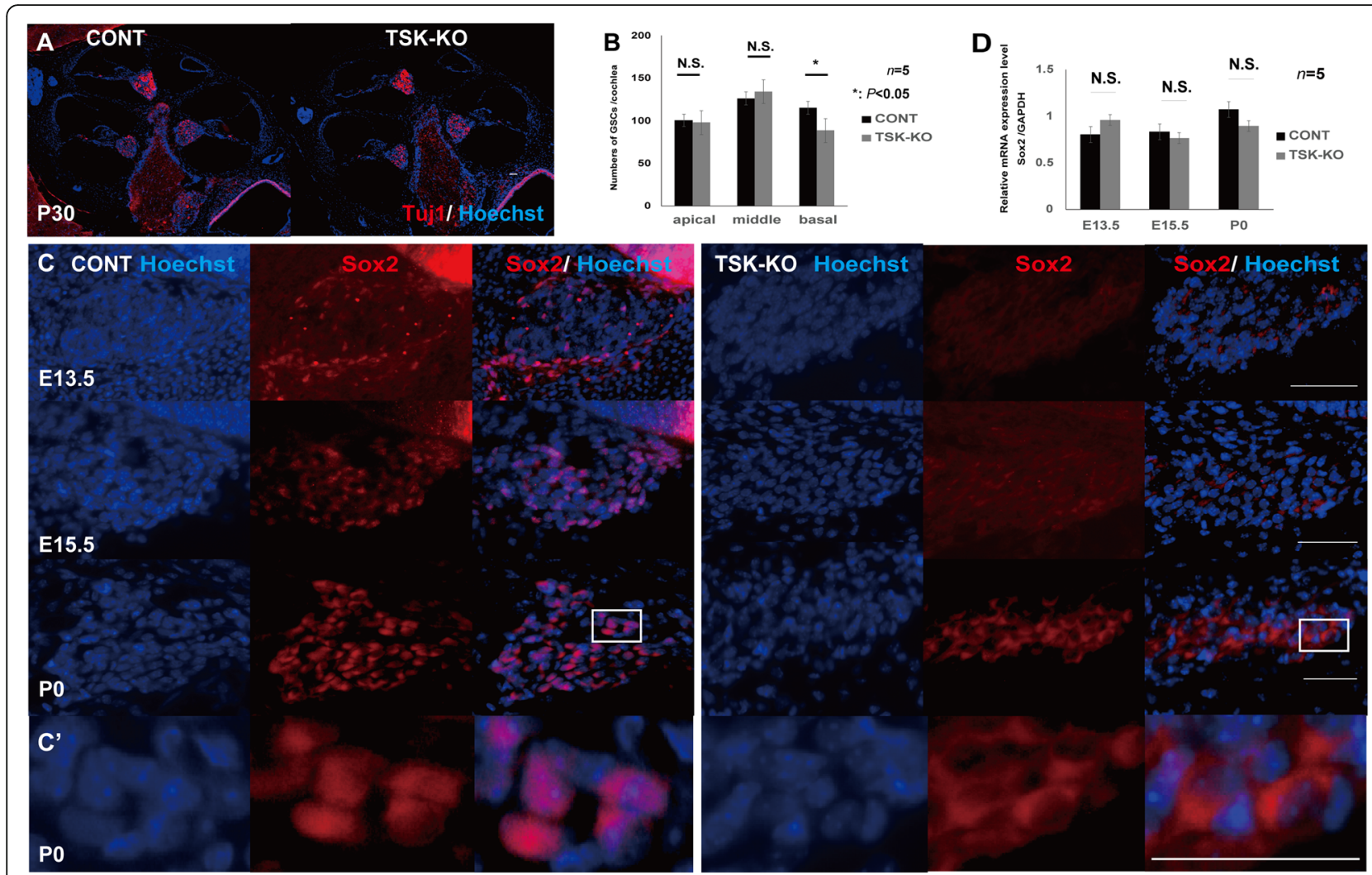

Fig. 5 Assessment of spiral ganglion cells in the CONT and TSK-KO mice. a Gross morphology. $\mathbf{b}$ Differences in numbers were not significant at apical $(p=0.39)$ and middle $(p=0.15)$ turns, but were significant at basal turns ( $\left.{ }^{*}, p=0.02\right)$. c Immunolabeling showed that Sox2 accumulated in the nuclei of the spiral ganglion cells in the CONT mice but was suppressed in the TSK-KO mice at E13.5, E15.5, and P0. Sox2 translocated to the cytosol at PO in the TSK-KO mice. ( (') Magnified view of the white hollow square in C. $\mathbf{d}$ qRT-PCR for Sox2 mRNA in the spiral ganglion tissue collected by laser microdissection did not detect significant differences in Sox2 mRNA between the CONT and TSK-KO mice. $p=0.09$ at E13.5; $p=0.34$ at E15.5; $p=0.21$ at P0. N.S.: not significant

\section{TSK deficiency translocated Sox2 in the spiral ganglion cells}

Immunolabeling showed that Sox2 accumulated in the nuclei of the SGCs in the CONT mice but was suppressed in the TSK-KO mice at E13.5, E15.5, and P0 (Fig. 5c). Interestingly, Sox 2 translocated to the cytosol at $\mathrm{PO}$ in the TSK-KO mice (Fig. 5c) only at the basal turn. In addition, qRT-PCR did not detect significant differences in Sox 2 mRNA between the CONT and TSK-KO mice (Fig. 5d), with $p=0.09,0.34$, and 0.21 at E13.5, E15.5, and P0, respectively.

TSK loss did not affect the stria vascularis, spiral ligament, and endocochlear potentials

Thickness of the stria vascularis (Fig. 6a, b, $p=0.13$ ) and expression of claudin-11 and the potassium channel KCNQ1, which are markers of the marginal and basal cells, respectively, did not differ between the CONT and TSK-KO mice. Endocochlear potentials decreased in the TSK-KO mice, but only marginally (Fig. $6 c, p=0.20$ ). In addition, expression of connexion-26 (Cx26), a marker of the spiral ligament, was comparable between the CONT and TSK-KO mice except for the subcentral region below the spiral prominence (Fig. 6d).

\section{Discussion}

The molecular mechanisms underlying cochlear development to achieve normal hearing have been challenging to elucidate [11]. Since TSK was previously shown as an organizer inducer that inhibits BMP [3], we investigated its roles in the differentiation of HCs, SGCs, and the stria vascularis. Our results suggested that the main pathomechanism of hearing loss in TSK-KO mice was the shortening of stereocilia of inner $\mathrm{HCs}$, caused by BMP gradient expression due to TSK deficiency (Additional file 3).

\section{TSK is required for hearing and stereocilia generation in} the inner hair cells

We detected TSK in the prosensory and nonprosensory regions of the cochlear epithelial cells of embryonic and adult mice. Further, we found that TSK deficiency in the developing embryonic inner ear 

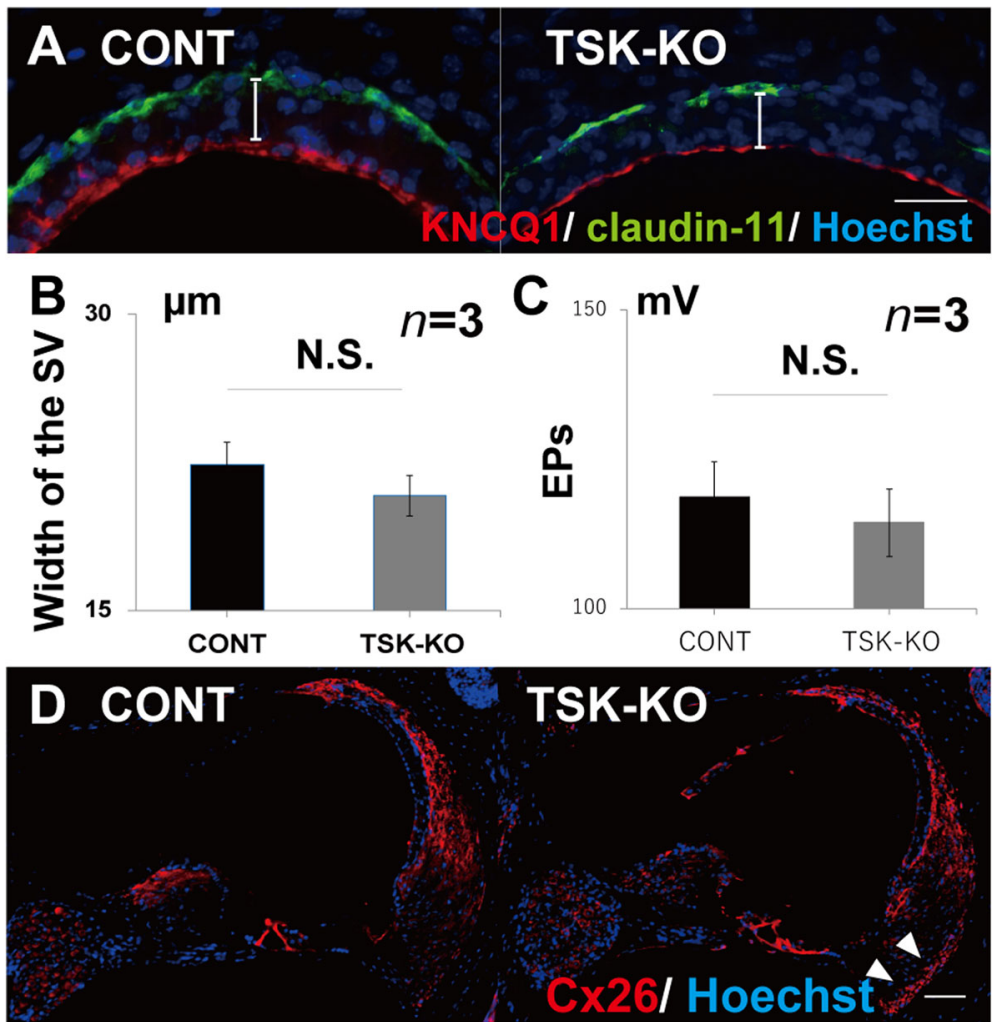

Fig. 6 Assessment of the stria vascularis in the CONT and TSK-KO mice. $\mathbf{a}$, $\mathbf{b}$ Thickness of the stria vascularis (SV) ( $p=0.13)$ and expression of claudin-11 and the potassium channel KCNQ1, which are markers of the marginal and basal cells, did not differ between the CONT and TSK-KO mice. c Endocochlear potentials (EPS) decreased in the TSK-KO mice, but only marginally $(p=0.20)$. $\mathbf{d}$ In addition, expression of connexion-26 (Cx26), a marker of the spiral ligament, was comparable between the CONT and TSK-KO mice except for the subcentral region below the spiral prominence. N.S.: not significant

redistributed BMP4 to a thin layer throughout the cochlear epithelium, resulting in BMP4 depletion from the outer sulcus and slight accumulation of BMP4 in prosensory regions. Thus, TSK deficiency appears to dispel an inherently asymmetric BMP signaling gradient, which is reportedly critical to inducing and patterning sensory domains in the mammalian cochlea [13]. In addition, loss of BMP signaling in the developing inner ear reportedly causes marked but "incomplete" loss of HCs [12]. We observed that stereocilia in the inner $\mathrm{HCs}$ were shortened in the TSK-KO mice, with no loss of the inner or outer HCs. These observations may explain why TSK-KO mice suffer hearing loss as young adults, without loss of the outer HCs.

Moreover, TSK loss suppressed Sox 2 at the prosensory regions that differentiate into the OC. Sox2 upregulates the transcription factor Atoh1 in both embryonic and postnatal cochlear progenitor cells, thus triggering differentiation of the HCs [14-17]. Indeed, the inner HCs are markedly susceptible to Atoh1, which controls the formation and organization of the stereocilia in a dosedependent manner $[18,19]$. Further, a stable and precise level of Atoh1 is needed for the development and maintenance of different HCs [20], possibly through the regulation of many essential downstream genes [21]. Previous studies also suggested that the level and duration of Sox2, FGF8, or miR-96 expression impact stereocilia formation, neuronal segregation, and the final HC phenotype, including their number and size $[18,22]$. For example, Sox2 deletion at later stages of the embryonic inner ear development elicits BMP4 expression adjacent to the greater epithelial ridge instead of lateral to the $\mathrm{OC}$, as well as the formation of ectopic inner $\mathrm{HCs}$ at the boundary of the greater epithelial ridge and the BMP4-expressing tissue [23]. As a corollary, BMP is known to repress Sox2 post-transcriptionally, as was observed during cardiac development [24].

Therefore, we believe that redistribution of BMP4 to the entire cochlear epithelium and Sox2 loss from prosensory regions cause abnormal development of the sensory domain in the cochlea, specifically shortening of the stereocilia in the inner HCs. However, our present study has not described how early changes in BMP4 or Sox2 are related to the P30 phenotypes; therefore, further research is necessary for the changes during postnatal development. 
TSK signaling is required to generate spiral ganglion cells Primary auditory neurons, also known as spiral ganglion neurons, receive chemical signals from the cochlear $\mathrm{HCs}$ and transmit information to the central cochlear nucleus in the brainstem [25]. These primary auditory neurons differentiate from neuronal precursors that delaminate from the otocyst early during the inner ear development [26]. A number of transcription factors, including Neurog1 [27], NeuroD1 [28], GATA3 [29], and Sox2 [16, 30], have been identified to drive neurogenesis and specification of the primary auditory neurons. Expansion and maintenance of neuronal precursors depend on Sox2 [23, 31]. Accordingly, Sox2 expression is downregulated early during embryonic development to allow neurogenesis via Neurog1 and NeuroD1 [32] but is then upregulated in the primary auditory neurons around birth. Our data show that in the absence of TSK, Sox2 is translocated from the nucleus to the cytoplasm at later embryonic stages only at the basal turn. Consequently, fewer SGCs were observed at the basal turn in the adult TSK-KO than in the CONT mice at P30. As the development of the SGCs is known to occur from the basal to the apical turn, we believe that TSK accumulates first in the basal turn as well, followed by Sox 2 expression during later embryonic stages. Therefore, Sox2 deficiency at the basal turn due to loss of TSK may explain the decrease in the number of SGCs. Sox 2 expression was decreased in the cochlear epithelium of TSK-KO mice due to BMP4 redistribution under TSK deficiency, whereas it was not decreased in SGCs but translated to the cytosol. We speculated that TSK deficiency directly affected Sox2. However, further research is needed to establish whether temporal sweep in the change of localization of Sox 2 to the developing SGCs is affected in the mutant.

\section{TSK signaling is not required to generate endocochlear potentials}

In the lateral wall, TSK expression dramatically redistributes from primarily the stria vascularis and type I fibrocytes of the spiral ligament to the stria vascularis and to type III fibrocytes of the spiral ligament. TSK deficiency causes Cx26 expression in type III fibrocytes of the spiral ligament. However, our data do not link TSK deficiency to changes in the morphology of the stria vascularis and spiral ligament, nor to endocochlear potentials. Hence, further research is necessary to clarify these aspects.

\section{TSK deficiency caused hearing loss but did not impair learning and memory}

The results of cochlear morphology assessment, increased ABR at all frequencies, normal DPOAE, and reduced number of SGCs at the basal turn in the TSK-KO mice suggested that shortening of stereocilia of inner $\mathrm{HCs}$ was the main pathomechanism of hearing loss in TSK-KO mice. However, there was a possibility of central hearing loss due to cognitive dysfunction. Therefore, we investigated cognitive function using the fearconditioning test to clarify hearing loss and to examine learning and memory function in the TSK-KO mice. Our results suggest that TSK-KO mice present with hearing loss but do not suffer any impairment of learning and memory function.

The main limitations of our study are as follows. First, we could not explain whether early changes in BMP4 or Sox2 were related to the P30 phenotypes. Second, the relation between TSK deficiency and morphological changes in the stria vascularis and spiral ligament, could not be clarified. Third, we could not establish whether temporal sweep in the change of localization of Sox 2 to the developing SGCs was related to TSK deficiency. Further studies addressing these limitations are recommended.

\section{Materials and methods \\ TSK-knockout (KO) mice}

TSK-KO C57BL/6 J mice (Mus musculus domesticus) were generated by inserting a LacZ/Neo cassette into the TSK-coding exon, as described previously [8]. Wildtype littermates by genotyping were used as CONT. Heterozygous littermates by genotyping were used for LacZ expression experiments. Mice were housed in an airconditioned room, maintained at about $25^{\circ} \mathrm{C}$ and $50 \%$ humidity. Standard commercial pellet diet and water were available ad libitum. The day at which vaginal plug was observed was designated as E0.5 at noon.

\section{Tissue processing}

Embryo heads were dissected and fixed in 4\% paraformaldehyde (PFA) in phosphate-buffered saline (PBS) for $12 \mathrm{~h}$ at $4{ }^{\circ} \mathrm{C}$. For postnatal assessments, mice were anesthetized by intraperitoneal injection of $4 \mathrm{mg} / \mathrm{kg}$ xylazine (Bayer, Shawnee Mission, KS, USA) and $120 \mathrm{mg} / \mathrm{kg}$ ketamine-HCl (Daiichi Sankyo, Tokyo, Japan) in 0.9\% $\mathrm{NaCl}$, and fixed by cardiac perfusion of $4 \%$ PFA in PBS. Subsequently, inner ears were dissected from temporal bones and decalcified in $0.5 \mathrm{M}$ EDTA (Wako, Osaka, Japan) for 3 days at $25^{\circ} \mathrm{C}$. Cochleae were embedded in OCT (Sakura Finetek Japan, Tokyo, Japan) and serially sectioned at $12 \mu \mathrm{m}$ thickness by a cryostat.

\section{$\beta$-Galactosidase staining}

TSK expression was investigated throughout the developmental stages of the inner ear by staining for LacZ in mice heterozygous for LacZ insertion in TSK. In particular, the inner ear was examined at embryonic stages E9.5, E11.5, E13.5, and E15.5, as well as at P0, P10, and P30 (each stage, $n=5$ ). Slides were dried for $30 \mathrm{~min}$ at 
$25^{\circ} \mathrm{C}$, stained with $400 \mathrm{mg} / \mathrm{mL}$ X-gal (Genlantis, San Diego, CA, USA) for $18 \mathrm{~h}$ at $37^{\circ} \mathrm{C}$, and imaged at $1360 \times 1024$ pixels using a BZ-9000 microscope (Keyence, Osaka, Japan) using uniform photographic exposure parameters.

\section{Immunostaining}

Sections were blocked with $10 \%(\mathrm{v} / \mathrm{v})$ normal goat serum in PBS containing $0.1 \%$ Triton X-100 (IBI Scientific, Peosta, IA, USA) for $10 \mathrm{~min}$ at $25^{\circ} \mathrm{C}$ and labeled using rabbit antibody against acetylated $\alpha$-tubulin (Tuj1; 1:500, COVANCE, Princeton, NJ, USA) and mouse antibody against Sox2 (1:200, Cell Signaling, CA, USA) in a humidified chamber for $1 \mathrm{~h}$ at $25^{\circ} \mathrm{C}$. Subsequently, sections were washed and incubated with goat anti-mouse or anti-rabbit IgG conjugated to Alexa 594 (1:500, Thermo Fisher Scientific, Rockford, IL, USA) for $1 \mathrm{~h}$ at $25^{\circ} \mathrm{C}$. After washing with PBS, tissue sections were counterstained with Hoechst 33342 (Molecular Probes, OR, USA), mounted, covered with coverslips, and imaged at $1360 \times 1024$ pixels by using a BZ-9000 fluorescence microscope (Keyence) with uniform photographic exposure parameters. Sections representative of at least five mice were presented.

\section{Enumeration of hair cells}

HCs were counted as described previously [33]. In brief, cochleae were removed from temporal bones at P0, P6, and P30 and fixed for $18 \mathrm{~h}$ at $4{ }^{\circ} \mathrm{C}$. Bony capsules and lateral walls were then removed. After blocking with 0.3\% Triton X-100 in PBS for 10 min, the organ of Corti (OC) was labeled using rabbit antibody against Myo7a (1:500, Thermo Scientific Pierce, PA1-936) and goat anti-mouse or anti-rabbit IgG conjugated to Alexa 488 (1:500, Thermo Fisher Scientific). Tissue sections were counterstained with Hoechst 33342 (Molecular Probes), mounted, and covered with coverslips for P30 cochleae, while the OC was stained by Texas Red-X phalloidin (Molecular Probes) for $30 \mathrm{~min}$ for P0, P6, and P30 cochleae (P0, each $n=2$; P6, each $n=2$; P30, each $n=5$ ). Between steps, tissue sections were washed with PBS thrice for $5 \mathrm{~min}$ each. Surface images of the OCs were captured, and the inner and outer $\mathrm{HCs}$ were counted after Myo7a staining over $300 \mu \mathrm{m}^{2}$ of tissue. The viability of HCs was also assessed (CONT and TSK-KO, each $n=5$ at P30).

\section{Enumeration of the spiral ganglion cells}

SGCs were counted as described previously [34]. Briefly, after immunostaining for Tuj1 and counterstaining with Hoechst, we delineated the Rosenthal's canal as the area that contains clusters of doubly stained cells and extends laterally to the modiolus. SGCs in the Rosenthal's canal, which were positive for both Tuj1 and Hoechst stain, were then marked and counted visually using ImageJ (NIH, Bethesda, MD, USA) at the apical, middle, and basal turns using three randomly selected sections per cochlea (CONT and TSK-KO, each $n=5)$. A second researcher reviewed the results for accuracy and calculated the average.

\section{Measurement of the thickness of the stria vascularis}

The average thickness of the stria vascularis was measured by analyzing images of the sections containing the midmodiolar region by using Image (NIH) (CONT and TSK-KO, each $n=3$ ).

\section{In situ hybridization}

Cochleae collected at E13.5, E15.5, and P0 were examined by in situ hybridization according to Moorman et al. [35], by using antisense riboprobes for BMP4 and Sox2 that were labeled with digoxigenin using DIG RNA Labeling Kit (Roche, Indianapolis, IN, USA). The probes were produced using the corresponding DNA constructs. Detailed protocols are available upon request. A minimum of five samples were prepared for each time point (CONT and TSK-KO, each $n=5)$.

\section{Scanning electron microscopy}

Each mouse was anesthetized as described above and briefly perfused with $0.9 \% \mathrm{NaCl}$, followed by $20 \mathrm{~mL}$ of $2.5 \%$ glutaraldehyde and $2 \mathrm{mM} \mathrm{CaCl}_{2}$ in $0.1 \mathrm{M}$ sodium cacodylate ( $\mathrm{pH} 7.4$ ), through the ascending aorta. The inner ear was removed by dissection. The top of the cochlea was punctured using a fine-tipped pair of forceps, and semicircular canals were sliced open. The inner ear was then gently flushed through these openings using $0.3 \mathrm{~mL}$ of $2.5 \%$ glutaraldehyde and subsequently fixed in the same solution for $18 \mathrm{~h}$ at $4{ }^{\circ} \mathrm{C}$. The membranous labyrinth containing the cochlea was removed by dissection. Cochlear specimens were prepared by removing the stria vascularis, Reissner's membrane, and tectorial membrane. The cochlear spiral was sectioned into basal, middle, and apical segments and stained with $1 \% \mathrm{OsO}_{4}$ thrice for $1 \mathrm{~h}$, based on the technique by Hunter-Duvar [36]. Samples were incubated with saturated thiocarbohydrazide for $20 \mathrm{~min}$ after the first and second $\mathrm{OsO}_{4}$ treatments. Specimens were then dehydrated in a graded series of ethanol and critical-point-dried using liquid $\mathrm{CO}_{2}$ as transitional fluid. Uncoated specimens were mounted on a Hitachi specimen stub, using silver electroconductive paint, and imaged using a Hitachi S-4800 field-emission scanning electron microscope operated at $5 \mathrm{kV}$. Five mice from each group (CONT and TSK$\mathrm{KO})$ were studied. 


\section{Quantification of stereocilia}

Stereociliary dimensions were measured by using ImageJ (NIH) as described previously [37]. Ten inner HCs per mouse were imaged at 20,000× magnification at basal, middle, and apical turns to measure the average length of the four tallest stereocilia in each $\mathrm{HC}$ (CONT and TSK-KO, each $n=5$ ).

\section{Laser microdissection}

At E13.5, E15.5, and P0, whole heads were dissected and incubated in $4 \%$ PFA for $1 \mathrm{~h}$ at $25^{\circ} \mathrm{C}$. Samples were then embedded in OCT, sectioned at $10 \mu \mathrm{m}$ in the plane of the long axis of the cochlear modiolus, mounted on uncharged slides (Leica Microsystems, Wetzlar, Germany), and dried at $25^{\circ} \mathrm{C}$. Slides were incubated in $95 \%$ acetone at $-20^{\circ} \mathrm{C}$ and dried at $25^{\circ} \mathrm{C}$ immediately before laser microdissection using an LMD7 system (Leica Microsystems), as described by Pagedar et al. [38]. Cell samples were obtained from cochlear epithelium or SGCs. Each slide contained multiple adjacent sections, and all cells in each category were pooled from individual slides onto a single cap (CONT and TSK-KO, each $n=5)$.

\section{Quantitative reverse-transcription polymerase chain reaction}

Using a microRNA extraction kit (QIAGEN, Valencia, CA, USA), total RNA was extracted from each sample obtained by laser microdissection, quantified using a GeneQuant100 (GE Healthcare, Amersham, UK), and diluted as needed to achieve uniform concentrations. cDNA was then synthesized using a One-Step PrimeScript RT-PCR Kit (Takara Bio, Otsu, Japan) according to the manufacturer's instructions, using primers for BMP4, Sox2, and GAPDH (Applied Bionics, Foster City, CA, USA). Targets were amplified using a Takara Dice TP960 over 40 cycles of denaturation at $95^{\circ} \mathrm{C}$ for $15 \mathrm{~s}$ and annealing at $60^{\circ} \mathrm{C}$ for $1 \mathrm{~min}$. Relative gene expression was calculated by generating a standard curve and normalized to GAPDH signal (CONT and TSK-KO, each $n=7$ ).

\section{Auditory function}

Mice were anesthetized at P30 as described above, and electrodes were placed beneath the pinna of the test ear and at the vertex just below the surface of the skin. The ground electrode was placed under the contralateral ear. Auditory thresholds were measured at 4, 12, and $20 \mathrm{kHz}$ by recording the $\mathrm{ABR}(15 \mathrm{~ms}$ duration, $1 \mathrm{~ms}$ rise/fall time, and tone burst) on a System 3 (Tucker-Davis Technologies, Alachua, FL, USA). For each recording, 1024 sweeps were averaged. Stimulus levels near the threshold were changed in 10-dB steps, and the threshold was defined as the lowest level at which waves in the
ABR were clearly detectable by visual inspection (CONT and TSK-KO, each $n=5$ ).

\section{Otoacoustic emissions}

Five mice from each group were anesthetized as described above, and pinnae were removed. An ER10B+ probe microphone/speaker system with two speaker ports (Etymotic Research, Inc., Elk Grove Village, IL, USA) was fitted tightly into the ear canal and linked to two closed-field EC-1 speakers (Tucker-Davis Technologies). Two primary tones were generated ( $1 \mathrm{~s}$ duration with $20 \mathrm{~ms}$ rise/fall cosine ramp; f $2 / \mathrm{f} 1=1.22$, f 2 varied at a one-fourth octave step from 4 to $29 \mathrm{kHz}$ ) and routed separately to the two EC-1 speakers at SPL1 $=75 \mathrm{~dB}$ and SPL2 $=65 \mathrm{~dB}$. The SPL was calibrated in a $0.1-\mathrm{mL}$ coupler [39] using a Brüel and Kjær 1/4" pressure field microphone (model 4938), which has a flat frequency response from $4 \mathrm{~Hz}$ to $70 \mathrm{kHz}$. The calibration was conducted for primary tones and all the components of the DPOAE. The DPOAE response from the ER10B+ microphone was amplified by $20 \mathrm{~dB}$ and digitized at $150 \mathrm{kHz}$ using a PCI-MIO-16E-1 A/D converter (National Instruments, Austin, TX, USA). Data were acquired and analyzed using customized software written in Matlab (The Mathworks, Natick, MA, USA). Recordings were repeated 10 times at 20-s intervals and averaged as a function of time. The noise was estimated by averaging three adjacent frequency bins that were above and below the DPOAE frequency [40].

\section{Endocochlear potentials}

Endocochlear potentials were recorded at P30 under general anesthesia [41]. The cochlea was firstly exposed ventrally. The bone over the spiral ligament was thinned, and a small opening was generated with a pick to access the endolymphatic compartment (scala media) of the basal turn. A heat-pulled micropipette electrode filled with 150 $\mathrm{mM} \mathrm{KCl}$ was inserted into this compartment until a stable potential was recorded, at which point potentials no longer depended on the electrode depth. The signal was amplified through an MEZ-7200 amplifier (Nihonkoden, Tokyo, Japan), and direct current potentials were recorded using a USB-6216 A/D converter (National Instruments) (CONT and TSK-KO, each $n=3$ ).

\section{Contextual and cued fear-conditioning test}

To assess fear-associated learning and memory, each mouse was placed in a test chamber $(33 \times 25 \times 28 \mathrm{~cm})$ with a stainless-steel grid floor $(0.2 \mathrm{~cm}$ diameter, spaced $0.5 \mathrm{~cm}$ apart; O'HARA \& CO., Tokyo, Japan) in a soundattenuating chamber to control environmental conditions and allowed to explore for $2 \mathrm{~min} \mathrm{[42].} \mathrm{Subse-}$ quently, a conditioned stimulus (CS; $55 \mathrm{~dB}$ white noise) was presented for $30 \mathrm{~s}$, followed by a mild foot shock (2 
s, $0.3 \mathrm{~mA}$ ), which served as the unconditioned stimulus (UCS). Two more CS-UCS pairs were presented at 2min interstimulus intervals. Context test was conducted 1 day after conditioning in the same chamber for $300 \mathrm{~s}$ on each mouse. A cued test with an altered context was then conducted in a triangular chamber $(33 \times 29 \times 32$ $\mathrm{cm})$ made of white opaque plastic, placed in a different room. Tone stimulus for the cued test $(55 \mathrm{~dB}$ white noise) was applied for $180 \mathrm{~s}$. In each test, freezing percentage was calculated automatically using the ImageFZ software based on the public domain Image J program and developed and modified by Tsuyoshi Miyakawa (available through O'HARA \& CO.) (CONT: $n=15$, TSK-KO: $n=17$ ).

\section{Experimental design and statistical analyses}

Data are reported as mean \pm s.d. Two groups were compared using Mann-Whitney $U$ test. For comparisons of more than two groups, one-way or two-way repeatedmeasures ANOVA was performed, followed by Bonferroni's post-hoc test of pairwise group differences. Statistical calculations were performed using Microsoft Excel (Redmond, WA, USA). Results with a $p$ value $<0.05$ were considered statistically significant.

\section{Supplementary information}

Supplementary information accompanies this paper at https://doi.org/10. 1186/s13041-020-00570-z.

Additional file 1: Behavioral and fear-conditioning tests in the CONT and TSK-KO mice. Freezing responses between the CONT and TSK-KO mice in the conditioning phase (auditory stimulus and foot shock, 2-min interstimulus interval) or contextual testing (1 day after conditioning for $300 \mathrm{~s}$ ) were not significantly different (conditioning phase, $p=0.94$; contextual testing, $p=0.75$ ). Levels of freezing in the TSK-KO mice significantly decreased during the auditory cue in an altered context (auditory stimulus for $180 \mathrm{~s})$ compared with that in the CONT mice $(p=0.02)$. CONT mice: $n=15$, TSK-KO mice: $n=17$. CS: conditioned stimulus, UCS: unconditioned stimulus, N.S.: not significant.

Additional file 2. Enumeration of hair cells in surface preparations of cochleae. Stereocilia of inner hair cells at P0 and P6 were likely to be shortened in the surface preparation. IHC: inner hair cell, OHC: outer hair cell.

Additional file 3. Schematic drawing of TSK function in the cochlea. (A) TSK function in cochlea epithelium from E13.5 to P30. (B) TSK function in the spiral ganglion from E18.5 to P30. WT: wild-type.

\section{Abbreviations}

ABR: Auditory brainstem response; CS: Conditioned stimulus; DPOAE: Distortion product of otoacoustic emission; EP: Endocochlear potential; HC: Hair cell; OC: Organ of corti; PBS : Phosphate-buffered saline; PFA : Paraformaldehyde; SGC : Spiral ganglion cell; TSK: Tsukushi; UCS : Unconditioned stimulus

\section{Acknowledgements}

Behavioral battery test was carried out at Institute for Comprehensive Medical Science, Fujita Health University (Joint Usage/Research Center for Genes, Brain and Behavior) and was supported by a Grant-in-Aid for Scientific Research on Innovative Areas "Platform of Advanced Animal Model Support" (16H06276) from the Ministry of Education, Science, Sports and Culture of
Japan. We thank Ayako Ito, Miho Kataoka, Momoko Ise, and Kyoko Tachii for technical assistance and all members of our labs for valuable help.

\section{Authors' contributions}

TM, SH, and WJS performed experiments. TM analyzed data and wrote the manuscript. KO, NI, TsM, TT, NN, WJS, and RM designed the experiments and supervised the project. The author(s) read and approved the final manuscript.

\section{Funding}

This research received no specific grant from any funding agency in the public, commercial, or not-for-profit sectors.

\section{Availability of data and materials}

The datasets used and/or analyzed during the current study are available from the corresponding author on reasonable request.

Ethics approval and consent to participate

Experiments were approved by the Committee on the Use and Care of Animals at Kumamoto University (Number H28-053) and Fujita Health University (Number AP16016) and complied with veterinary standards.

Consent for publication

Not applicable.

\section{Competing interests}

The authors declare that they have no competing interests.

\section{Author details}

${ }^{1}$ Department of Otolaryngology-Head and Neck Surgery, Kitano Hospital, Tazuke Kofukai Medical Research Institute, 2-4-20 Ougimaci, Kita-ku, Osaka 5308084, Japan. ²Department of Otolaryngology-Head and Neck Surgery, Graduate School of Medicine, Kyoto University, 54 Shogoin Kawahara-cho, Sakyo-ku, Kyoto 6068507, Japan. ${ }^{3}$ Departments of Otolaryngology-Head and Neck Surgery, Graduate School of Medicine, Kumamoto University, 1-1-1 Honjo, Kumamoto 8608556, Japan. ${ }^{4}$ Otolaryngology-Head and Neck Surgery, JCHO Kumamoto General Hospital, 10-10 Toricho, Yatsushiro 8668660, Japan. ${ }^{5}$ Department of Developmental Neurobiology, Graduate School of Life Sciences, Kumamoto University, 1-1-1 Honjo, Kumamoto 8608556, Japan. ${ }^{6}$ Program for Leading Graduate Schools HIGO Program, Kumamoto University, 2-2-1 Honjo, Kumamoto 8608556, Japan. ${ }^{7}$ Global COE Cell Fate Regulation Research and Education Unit, Kumamoto University, 2-2-1 Honjo, Kumamoto 8600881, Japan. ${ }^{8}$ Japan Agency for Medical Research and Development (AMED), Tokyo 1000004, Japan. ${ }^{9}$ Division of Systems Medical Science, Institute for Comprehensive Medical Science, Fujita Health University, 1-98 Dengakugakubo, Kutsukak, Toyoake 4701192, Japan. ${ }^{10}$ Division of Reproductive Engineering, Center for Animal Resources and Development (CARD), Kumamoto University, 2-2-1 Honjo, Kumamoto 8600881, Japan. ${ }^{11}$ Department of Sensory and Cognitive Physiology, Graduate School of Life Sciences, Kumamoto University, 1-1-1 Honjo, Kumamoto 8608556, Japan.

Received: 27 December 2019 Accepted: 20 February 2020 Published online: 03 March 2020

\section{References}

1. Ahmad SAI, Anam MB, Ito N, Ohta K. Involvement of Tsukushi in diverse developmental processes. J Cell Commun Signal. 2018;12:205-10.

2. Dellett M, Hu W, Papadaki V, Ohnuma S. Small leucine rich proteoglycan family regulates multiple signaling pathways in neural development and maintenance. Develop Growth Differ. 2012;54:327-40.

3. Ohta K, Lupo G, Kuriyama S, Keynes R, Holt CE, Harris WA, et al. Tsukushi functions as an organizer inducer by inhibition of BMP activity in cooperation with chordin. Dev Cell. 2004;7:347-58.

4. Hocking AM, Shinomura T, McQuillan DJ. Leucine-rich repeat glycoproteins of the extracellular matrix. Matrix Biol. 1998;17:1-19.

5. Merline R, Schaefer RM, Schaefer $L$. The matricellular functions of small leucine-rich proteoglycans (SLRPS). J Cell Commun Signal. 2009;3:323-35.

6. Schaefer $L$, lozzo RV. Biological functions of the small leucine-rich proteoglycans: from genetics to signal transduction. J Biol Chem. 2008;283: 21305-9. 
7. Hossain M, Ahmed G, Bin Naser I, Shinmyo Y, Ito A, Asrafuzzaman Riyadh M, et al. The combinatorial guidance activities of draxin and Tsukushi are essential for forebrain commissure formation. Dev Biol. 2013;374:58-70.

8. Ito A, Shinmyo Y, Abe T, Oshima N, Tanaka H, Ohta K. Tsukushi is required for anterior commissure formation in mouse brain. Biochem Biophys Res Commun. 2010:402:813-8.

9. Niimori D, Kawano R, Felemban A, Niimori-Kita K, Tanaka H, Ihn H, et al. Tsukushi controls the hair cycle by regulating TGF- $\beta 1$ signaling. Dev Biol. 2012;372:81-7.

10. Ohta K, Kuriyama S, Okafuji T, Gejima R, Ohnuma S, Tanaka H. Tsukushi cooperates with VG1 to induce primitive streak and Hensen's node formation in the chick embryo. Development. 2006;133:3777-86.

11. Heller S. Special issue on inner ear development and regeneration. Hear Res. 2013;297:1-2.

12. Li H, Corrales CE, Wang Z, Zhao Y, Wang Y, Liu H, et al. BMP4 signaling is involved in the generation of inner ear sensory epithelia. BMC Dev Biol. 2005:5:16.

13. Ohyama T, Basch ML, Mishina Y, Lyons KM, Segil N, Groves AK. BMP signaling is necessary for patterning the sensory and nonsensory regions of the developing mammalian cochlea. J Neurosci. 2010;30:15044-51.

14. Dabdoub A, Puligilla C, Jones JM, Fritzsch B, Cheah KSE, Pevny LH, et al. Sox2 signaling in prosensory domain specification and subsequent hair cell differentiation in the developing cochlea. Proc Natl Acad Sci U S A. 2008; 105:18396-401.

15. Kempfle JS, Turban JL, Edge ASB. Sox2 in the differentiation of cochlear progenitor cells. Sci Rep. 2016;6:23293.

16. Puligilla C, Dabdoub A, Brenowitz SD, Kelley MW. Sox2 induces neuronal formation in the developing mammalian cochlea. J Neurosci. 2010;30:714-22.

17. Zheng JL, Gao W-Q. Overexpression of Math1 induces robust production of extra hair cells in postnatal rat inner ears. Nat Neurosci. 2000;3:580-6.

18. Jahan I, Pan N, Kersigo J, Fritzsch B. Beyond generalized hair cells: molecular cues for hair cell types. Hear Res. 2013;297:30-41.

19. Jahan I, Pan N, Kersigo J, Fritzsch B. Neurog1 can partially substitute for Atoh1 function in hair cell differentiation and maintenance during organ of Corti development. Development. 2015;142:2810-21.

20. Pan N, Jahan I, Kersigo J, Duncan JS, Kopecky B, Fritzsch B. A novel Atoh1 "self-terminating" mouse model reveals the necessity of proper Atoh1 level and duration for hair cell differentiation and viability. PLoS One. 2012;7: e30358

21. Klisch TJ, Xi Y, Flora A, Wang L, Li W, Zoghbi HY. In vivo Atoh1 targetome reveals how a proneural transcription factor regulates cerebellar development. Proc Natl Acad Sci U S A. 2011;108:3288-93.

22. Pan W, Jin Y, Chen J, Rottier RJ, Steel KP, Kiernan AE. Ectopic expression of activated notch or SOX2 reveals similar and unique roles in the development of the sensory cell progenitors in the mammalian inner ear. J Neurosci. 2013;33:16146-57.

23. Dvorakova M, Jahan I, Macova I, Chumak T, Bohuslavova R, Syka J, et al. Incomplete and delayed Sox2 deletion defines residual ear neurosensory development and maintenance. Sci Rep. 2016;6:38253.

24. Rao J, Pfeiffer MJ, Frank S, Adachi K, Piccini I, Quaranta R, et al. Stepwise clearance of repressive roadblocks drives cardiac induction in human ESCs. Cell Stem Cell. 2016;18:341-53.

25. Dabdoub A. In: Fritzsch B, Popper AN, Fay RR, editors. The primary auditory neurons of the mammalian cochlea. New York: Springer; 2016.

26. Rubel EW, Fritzsch B. Auditory system development: primary auditory neurons and their targets. Annu Rev Neurosci. 2002;25:51-101.

27. Ma Q, Anderson DJ, Fritzsch B. Neurogenin 1 null mutant ears develop fewer, morphologically normal hair cells in smaller sensory epithelia devoid of innervation. J Assoc Res Otolaryngol. 2000;1:129-43.

28. Kim WY, Fritzsch B, Serls A, Bakel LA, Huang EJ, Reichardt LF, et al. NeuroDnull mice are deaf due to a severe loss of the inner ear sensory neurons during development. Development. 2001;128:417-26.

29. Lawoko-Kerali G, Rivolta MN, Lawlor P, Cacciabue-Rivolta DI, Langton-Hewer C, Holley MC, et al. GATA3 and NeuroD distinguish auditory and vestibular neurons during development of the mammalian inner ear. Mech Dev. 2004; 121:287-99.

30. Nishimura K, Noda T, Dabdoub A. Dynamic expression of Sox2, Gata3, and Prox 1 during primary auditory neuron development in the mammalian cochlea. PLOS One. 2017;12:e0170568.

31. Zhang S, Cui W. Sox2, a key factor in the regulation of pluripotency and neural differentiation. World J Stem Cells. 2014;6:305-11.
32. Evsen L, Sugahara S, Uchikawa M, Kondoh H, Wu DK. Progression of neurogenesis in the inner ear requires inhibition of Sox2 transcription by neurogenin1 and neurod1. J Neurosci. 2013;33:3879-90.

33. Takeda H, Kurioka T, Kaitsuka T, Tomizawa K, Matsunobu T, Hakim F, et al. Protein transduction therapy into cochleae via the round window niche in Guinea pigs. Mol Ther Methods Clin Dev. 2016;3:16055.

34. Yamada T, Minoda R, Miwa T, Ise M, Takeda H, Yumoto E. Neurogenesis of the spiral ganglion cells in the cochlea requires the transcriptional cofactor TIS21. Neurosci Lett. 2015:584:265-9.

35. Moorman AFM, Houweling AC, de Boer PAJ, Christoffels VM. Sensitive nonradioactive detection of mRNA in tissue sections: novel application of the whole-mount in situ hybridization protocol. J Histochem Cytochem. 2001:49:1-8.

36. Hunter-Duvar IM. A technique for preparation of cochlear specimens for assessment with the scanning electron microscope. Acta Otolaryngol Suppl. 1978;351:3-23

37. Sekerková G, Richter C-P, Bartles JR. Roles of the espin actin-bundling proteins in the morphogenesis and stabilization of hair cell stereocilia revealed in CBA/CaJ congenic jerker mice. PLoS Genet. 2011;7:e1002032.

38. Pagedar NA, Wang W, Chen DH-C, Davis RR, Lopez I, Wright CG, et al. Gene expression analysis of distinct populations of cells isolated from mouse and human inner ear FFPE tissue using laser capture microdissection - a technical report based on preliminary findings. Brain Res. 2006;1091:289-99.

39. Polesskaya O, Cunningham LL, Francis SP, Luebke AE, Zhu X, Collins D, et al. Ablation of mixed lineage kinase 3 (Mlk3) does not inhibit ototoxicity induced by acoustic trauma or aminoglycoside exposure. Hear Res. 2010; 270:21-7.

40. Lu M-H, Takemoto M, Watanabe K, Luo H, Nishimura M, Yano M, et al. Deficiency of sphingomyelin synthase-1 but not sphingomyelin synthase-2 causes hearing impairments in mice. J Physiol. 2012;590:4029-44.

41. Miwa T, Minoda R, Ise M, Yamada T, Yumoto E. Mouse otocyst transuterine gene transfer restores hearing in mice with connexin 30 deletion-associated hearing loss. Mol Ther. 2013:21:1142-50.

42. Shoji H, Takao K, Hattori S, Miyakawa T. Contextual and cued fear conditioning test using a video analyzing system in mice. J Vis Exp. 2014;85: e50871.

\section{Publisher's Note}

Springer Nature remains neutral with regard to jurisdictional claims in published maps and institutional affiliations.

\section{Ready to submit your research? Choose BMC and benefit from:}

- fast, convenient online submission

- thorough peer review by experienced researchers in your field

- rapid publication on acceptance

- support for research data, including large and complex data types

- gold Open Access which fosters wider collaboration and increased citations

- maximum visibility for your research: over $100 \mathrm{M}$ website views per year

At $\mathrm{BMC}$, research is always in progress.

Learn more biomedcentral.com/submission 